\title{
Turismo Rural Comunitario, Una Alternativa Para El Desarrollo De Los Territorios Rurales Estudio De Caso: Guasuntos, Ecuador
}

\author{
Mónica Cecilia Zurita Vintimilla \\ Gabriela Estefanía Román Santamaría \\ Gabriela Natalí Fonseca Romero \\ Luis Alberto Quevedo Báez \\ Danny Daniel Castillo Vizuete
}

Docentes - Investigadores -

Escuela Superior Politécnica de Chimborazo Riobamba, Ecuador

doi: 10.19044/esj.2017.v13n17p40 URL:http://dx.doi.org/10.19044/esj.2017.v13n17p40

\section{Abstract}

Ecuador is a country with a vast amount of natural and cultural heritage. The diversity of its four regions has allowed the presence of several species of flora and fauna. Most of its fauna and flora is present at 26 protected natural areas. Also, it offers historical attractions, gastronomy, and a variety of cultures and traditions. In all these aspects, Ecuador has developed several modalities of tourism, as the community based tourism, which is considered as a strategy for development. It is also considered as an object of investigation which includes three fundamental perspectives: special sensitivity to the natural environment and cultural particularities, integral sustainability (social and natural), and effective control of tourism by communities. Community tourism contributes to the country's brand at international level, being an element of growing interest in touristic market. The Guasuntos community has natural and cultural resources, which has allowed the incursion in this tourism modality. This, however, was possible through the creation of a product according to the needs of unsatisfied demand. A survey was applied to the local tourists who visited Alausí and Chunchi cantons. Also, the market analysis of the survey was carried out, and the potential demand and competition were projected. This helps to ascertain the unsatisfied demand in order to identify the needs of the national tourists. This study was developed as a contribution to the design of a touristic product with the aim of improving the population life quality. 
Keywords: Community tourism, unsatisfied demand, competition, product, productivity

\section{Resumen}

Ecuador es un país con una amplia riqueza natural y cultural. La diversidad de sus cuatro regiones ha dado lugar a diversas especies de flora y fauna. La mayor parte de su fauna y flora es tangible en 26 áreas protegidas por el estado. Así mismo, ofrece atractivos históricos, gastronómicos y una variedad de culturas y tradiciones; gracias a esto el Ecuador desarrolla varias modalidades de turismo, entre ellas el turismo comunitario, consolidado como una estrategia de desarrollo y objeto de investigación, que aúna tres perspectivas fundamentales: una sensibilidad especial con el entorno natural y las particularidades culturales, la búsqueda de sostenibilidad integral (social y natural) y el control efectivo del turismo por parte de las comunidades. El turismo comunitario se manifiesta como un importante contribuyente a la marca país a nivel internacional a través de un creciente interés en la oferta y demanda turística. La comunidad de Guasuntos cuenta con recursos naturales y culturales que le han permitido incursionar en esta modalidad de turismo, planteando la creación de un producto turístico acorde a las necesidades de la demanda insatisfecha, para lo cual se aplicó una encuesta a los turistas nacionales que visitaron el cantón Alausí y el cantón Chunchi, después se realizó el análisis de la competencia, y consecutivamente se proyectó la demanda potencial y la competencia, permitiendo así determinar la demanda insatisfecha a fin de identificar los gustos y necesidades de los turistas nacionales, con el objetivo de diseñar un producto turístico y fortalecer las cadenas de valor, con el enfoque de mejorar la calidad de vida de la población.

Palabras claves: Turismo comunitario, demanda insatisfecha, competencia, producto, productividad

\section{Introducción}

El turismo en Ecuador es la tercera actividad económica en importancia tras la extracción de petróleo y la producción de plátano, atrayendo a 700 mil visitantes al año (Ruiz, et al., 2008), es uno de los sectores priorizados para el cambio de la matriz productiva (MCPEYC, 2014). Su ubicación geográfica ha permitido al Ecuador estar dotado de 4 regiones: Galápagos, Costa, Andes y Amazonía (MAE, 2011), siendo así uno de los países con mayor biodiversidad del planeta, donde se pueden encontrar variedad de orquídeas, aves, mariposas, reptiles, anfibios y mamíferos (Mintur, 2009). 
El Ecuador cuenta con 14 nacionalidades y 18 pueblos indígenas (FEPTCE, 2010); culturalmente su potencialidad es apreciada en el idioma ancestral de la nacionalidad Zápara y la técnica de elaboración del sombrero de paja toquilla, declaradas como Patrimonio de la Humanidad por la UNESCO (2015). En este contexto el patrimonio natural y cultural refleja la biodiversidad, pluriculturalidad y multietnicidad que posee el país, siendo recursos que motivan la visitación turística, la conservación, y la gestión sostenible de los recursos.

En relación a la gestión se han desarrollado diversas actividades y modalidades en un sistema turístico integral (Torre, 2010), que permiten la dinamización de la economía a nivel nacional como herramienta estratégica para el desarrollo. La gestión se integra por procesos como la planeación, organización, dirección y control (Lledó, 2007), que al ser evidenciados en la actividad turística, reflejan la elasticidad entre la oferta y la demanda, funcionando como indicadores del comportamiento económico - social de la población, siendo un referente de evaluación en términos de productividad (Vázquez \& Damián, 2015).

Entre las acciones que el Ecuador ha desarrollado para que el turismo se genere de forma eficiente están: el Plan Nacional del Buen Vivir, enfocado en posicionar al país como un destino turístico de referencia a nivel mundial y el Plan Estratégico de Turismo (Plandetur, 2020), que contempla la estructura de productos y destinos turísticos, con objetivo de potencializar la matriz productiva a nivel nacional, provincial, cantonal y parroquial, reconociendo al turismo como uno de los sectores productivos prioritarios del país (Senplades, 2014).

El Plan Nacional del Buen Vivir o Sumak Kawsay, modelo de desarrollo al que el país responde, recoge una visión del mundo centrada en el ser humano, como parte de un entorno natural y social (Ministerio de Educación, 2011), ofreciéndole a la comunidad, oportunidades para que pueda desarrollar sus capacidades y potencialidades, se amplíe y florezca de modo que logre simultáneamente aquello que la sociedad, los territorios, las diversas identidades colectivas y cada uno deseen (Gudynas \& Acosta, 2011); ofrece alternativas para construir una sociedad más justa y supera los límites de las visiones convencionales de desarrollo, que lo conciben como un proceso lineal, de etapas históricas sucesivas, que reducen el concepto a una noción exclusiva de crecimiento económico (Senplades, 2014).

El turismo comunitario se convierte en una modalidad del turismo manejada a partir de las comunidades principalmente de la zona rural como un medio de reivindicación y autogestión sobre sus territorios y recursos (Ruiz et al., 2008), así, la iniciativa de turismo comunitario nace hace varios años en el Ecuador, a partir de las motivaciones propias de comunidades pioneras de la amazonia y la sierra (de la Cruz, 2007), esta forma de turismo 
favorece el contacto entre la comunidad local y los visitantes, obteniendo nuevas experiencias (Guzmán \& Cañizares, 2009). Las comunidades indígenas y campesinas ofertan turismo comunitario (TC), estimándose que esas actividades benefician directa e indirectamente a 15.000 personas (Ruiz et al., 2008).

La modalidad de turismo comunitario se presenta como una alternativa sostenible que contribuiría a los siguientes objetivos del Plan Nacional del "Buen Vivir": Objetivo 3 (Mejorar la calidad de vida de la población), Objetivo 4 (Fortalecer las capacidades y potencialidades de la ciudadanía), Objetivo 7 (Garantizar los derechos de la naturaleza y promover la sostenibilidad ambiental territorial y global), Objetivo 8 (Consolidar el sistema económico social y solidario, de forma sostenible), y el Objetivo 10 (Impulsar la transformación de la matriz productiva) (Senplades, 2014).

La parroquia Guasuntos ubicada en el cantón Alausí de la provincia de Chimborazo, posee recursos naturales y culturales de gran importancia que permiten desarrollar actividades de turismo y sus diversas modalidades, con el objetivo de generar alternativas de desarrollo para la población siempre en búsqueda de mejorar su calidad de vida, por lo cual el objetivo de este trabajo fue realizar un análisis de la demanda a partir de fuentes primarias, representadas en indicadores económicos y sociales, con el objetivo de identificar los gustos y preferencias de la demanda insatisfecha como mercado potencial para ofertar el turismo comunitario como una alternativa de desarrollo para la población.

\section{Metodología} documental.

Para la presente investigación se aplicaron técnicas de campo y

Inicialmente se realizaron talleres con la comunidad en la cual se trabajó la técnica de lluvia de ideas enfocadas en proponer iniciativas productivas a desarrollar en la "Playa de la Moya", a partir de esto se realizó una propuesta estructural del Complejo Turístico Playa la Moya; como siguiente paso se realizó un análisis de la demanda, identificando como universo de estudio el número total de turistas nacionales que visitaron el cantón Alausí en el año 2014 y el cantón Chunchi en el año 2012, correspondiente a 47460 turistas que visitaron el cantón Alausí según datos proporcionados por la Empresa Ferrocarriles del Ecuador y 2515 turistas que visitaron el cantón Chunchi según datos proporcionados por el departamento de Turismo del cantón. El cálculo de la muestra se realizó con un error del $8 \%$, valor de confianza igual a 2, probabilidad de ocurrencia y no ocurrencia de 0.5, obteniendo como resultado la aplicación de 119 encuestas a los turistas nacionales. 
El instrumento de investigación que fue aplicado para recolectar y obtener información fue la guía de entrevista (Anexo 1).

Posteriormente, se realizó el análisis y proyección de la competencia y la demanda potencial, utilizando el método de incremento compuesto cuyo modelo matemático es:

$$
C n=\operatorname{Co}(1+i) \mathrm{n} .
$$

Finalmente, se determinó la demanda insatisfecha calculando la diferencia entre la demanda potencial y la competencia.

\section{Resultados}

La parroquia de Guasuntos cuenta con un espacios natural representativo, la "Playa" en la comunidad la Moya, por lo cual, los pobladores del territorio han propuesto varias iniciativas a desarrollar en este espacio, que es el punto de encuentro de la comunidad y mantiene viva la historia y tradiciones de la población. Para identificar estas iniciativas se organizó un taller (Tabla 1) en el que se aplicó la técnica de "lluvia de ideas".

Tabla 1. Organización de la actividad "lluvia de ideas"

\begin{tabular}{|c|c|}
\hline Fecha: & Miércoles 12 de diciembre de 2016 \\
\hline Tiempo: & $08 \mathrm{~h} 30$ a $10 \mathrm{~h} 30$ \\
\hline Lugar: & Comunidad la Moya, Junta Parroquial \\
\hline Grupos de Enfoque: & Pobladores y autoridades de la comunidad. \\
\hline Tamaño del grupo: & $\begin{array}{l}\text { Dos grupos de } 10 \text { personas cada uno. Se considera un grupo } \\
\text { pequeño al trabajo inicial. }\end{array}$ \\
\hline Duración: & $\begin{array}{l}\text { 60-90 minutos (10m para motivar y colocar la técnica, 60m para } \\
\text { generar ideas y 20m para la evaluación). }\end{array}$ \\
\hline $\begin{array}{l}\text { Organización } \\
\text { Espacial: }\end{array}$ & $\begin{array}{l}\text { En la sala de reuniones de la Comunidad La Moya, Junta } \\
\text { Parroquial. }\end{array}$ \\
\hline Recursos necesarios: & Papelógrafos y marcadores. \\
\hline
\end{tabular}

Elaborado por: equipo de investigación

En el taller se realizó una explicación de cómo se trabajaría esta técnica y se motivó a la población en la generación de ideas (Tabla 2) con enfoque a desarrollar iniciativas de productividad.

Tabla 2. Resultado de la "lluvia de ideas"

\begin{tabular}{|c|c|}
\hline GRUPO A & GRUPO B \\
\hline Actividades productivas para desarrollar en la Playa de la comunidad La Moya \\
\hline Parque lineal & Área para deportes extremos \\
Área de conservación & Espacio para eventos (concha acústica) \\
Piscinas & Caballerizas \\
Lavanderías & Complejo turístico \\
Canoas & Comedores \\
Muelle & Canchas deportivas y vestidores \\
Escalada & Ciclismo \\
Estadio & Caminatas \\
Área de camping & Área de picnic \\
\hline
\end{tabular}

Elaborado por: equipo de investigación 
Después de la aplicación de la técnica de lluvia de ideas se filtró la información que dio como resultado el diseño del complejo turístico en el cual se integraron varias de las actividades que la población propuso como un aporte al desarrollo del turismo comunitario.

El análisis de la demanda permitió conocer el perfil del consumidor e identificar el nivel de aceptación de los diferentes emprendimientos y productos a desarrollarse en el complejo.

Partiendo de la encuesta aplicada a los turistas nacionales se definió el perfil del turista nacional (Tabla 3).

Tabla 3. Perfil del turista de origen nacional

\begin{tabular}{|c|c|c|c|}
\hline \multicolumn{4}{|c|}{ PERFIL DEL TURISTA } \\
\hline 1 & Género & $\begin{array}{l}\text { Masculino } \\
\text { Femenino }\end{array}$ & $\begin{array}{l}50 \% \\
50 \%\end{array}$ \\
\hline 2 & Edad & 36 - 45años & $34 \%$ \\
\hline 3 & Conoce Guasuntos & Si & $92 \%$ \\
\hline 4 & Visitar Guasuntos & $\mathrm{Si}$ & $83 \%$ \\
\hline 5 & Conoce la Playa La Moya & Si & $59 \%$ \\
\hline 6 & $\begin{array}{l}\text { Aceptación de implementación Complejo } \\
\text { Turístico }\end{array}$ & $\mathrm{Si}$ & $96 \%$ \\
\hline 7 & Fin de implementación del Complejo Turístico & $\begin{array}{l}\text { Recreativo } \\
\text { Ambiental }\end{array}$ & $\begin{array}{l}61 \% \\
39 \%\end{array}$ \\
\hline 8 & Actividades & $\begin{array}{l}\text { Ciclismo } \\
\text { Cabalgata } \\
\text { Camping } \\
\text { Escalada } \\
\text { Otras }\end{array}$ & $\begin{array}{l}23 \% \\
14 \% \\
18 \% \\
29 \% \\
16 \%\end{array}$ \\
\hline 9 & Espacios de encuentro común & $\begin{array}{c}\text { Concha acústica } \\
\text { Parqueadero } \\
\text { Comedor popular } \\
\text { Canchas deportivas } \\
\text { Espacios verdes } \\
\text { Otros }\end{array}$ & $\begin{array}{l}24 \% \\
20 \% \\
13 \% \\
16 \% \\
22 \% \\
05 \% \\
\end{array}$ \\
\hline 10 & Días de visita & $\begin{array}{c}\text { Domingo } \\
\text { Sábado } \\
\text { Viernes }\end{array}$ & $\begin{array}{l}46 \% \\
31 \% \\
23 \%\end{array}$ \\
\hline 11 & Horarios de visita & $8 \mathrm{am}-6 \mathrm{pm}$ & $38 \%$ \\
\hline 12 & Pago costo mínimo de ingreso & $\mathrm{Si}$ & $67 \%$ \\
\hline 13 & Medio de publicidad & $\begin{array}{c}\text { Internet } \\
\text { Material publicitario } \\
\text { impreso } \\
\text { Radio } \\
\text { Televisión } \\
\end{array}$ & $\begin{array}{l}42 \% \\
32 \% \\
21 \% \\
05 \%\end{array}$ \\
\hline
\end{tabular}

Elaborado por: equipo de investigación

\section{Análisis De La Competencia}

Se consideró como competencia a los lugares turísticos que tienen características similares al proyecto propuesto, sin embargo, no se identificó 
un proyecto similar, pero se tomó en cuenta la competencia complementaria y se consideró el centro de turismo comunitario de Tolte Pistishí (Tabla 4).

Tabla 4. Competencia complementaria

\begin{tabular}{|c|c|c|}
\hline \multicolumn{3}{|c|}{ COMPETENCIA } \\
\hline NOMBRE & CATEGORÍA & SERVICIOS QUE OFERTA \\
\hline & & Alimentación \\
\cline { 2 - 2 } Centro de Turismo & & Cabalgata \\
Comunitario Tolte & Centro de Turismo & Sendero \\
\cline { 2 - 2 } Pistishí Nariz del & Comunitario & Biking \\
Diablo. & & Fotografía \\
\cline { 3 - 3 } & & Granjas \\
\cline { 3 - 3 } & &
\end{tabular}

Elaborado por: equipo de investigación

\section{Demanda Insatisfecha}

Para el año 2017 la demanda insatisfecha será de 48744 turistas y se incrementará en el año 2021 a 51939 turistas (Tabla 5).

Tabla 5. Proyección de la demanda insatisfecha

\begin{tabular}{|c|c|c|c|c|}
\hline \multicolumn{5}{|c|}{ PROYECCIÓN DEMANDA INSATISFECHA } \\
\hline $\mathrm{N}^{\mathrm{o}}$ & AÑO & $\begin{array}{c}\text { DEMANDA } \\
\text { POTENCIAL }\end{array}$ & COMPETENCIA & $\begin{array}{c}\text { DEMANDA } \\
\text { INSATISFECHA } \\
\text { TOTAL }\end{array}$ \\
\hline 0 & 2016 & 47976 & 0 & 47976 \\
\hline 1 & 2017 & 48744 & 0 & 48744 \\
\hline 2 & 2018 & 49524 & 0 & 49524 \\
\hline 3 & 2019 & 50316 & 0 & 50316 \\
\hline 4 & 2020 & 51121 & 0 & 51121 \\
\hline 5 & 2021 & 51939 & 0 & 51939 \\
\hline
\end{tabular}

Elaborado por: equipo de investigación

Identificar la demanda insatisfecha permitió conocer los gustos y necesidades de los turistas nacionales y conjugarlo con el aporte de la comunidad para el diseño (Imagen 1) del complejo turístico con los diferentes emprendimientos seleccionados (Tabla 6).

Imagen 1. Playa la Moya

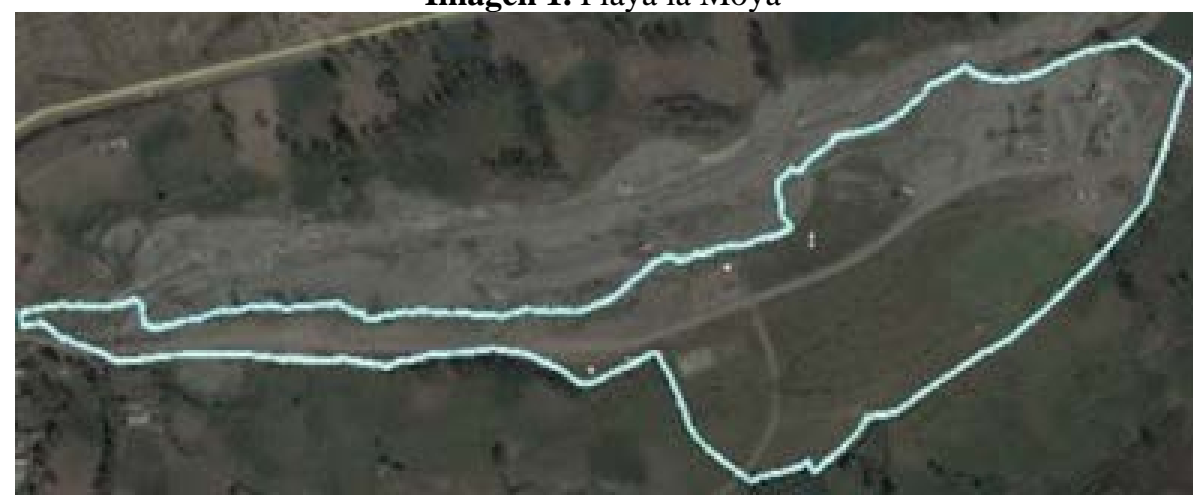

Elaborado por el equipo de investigación 
Imagen 2. Diseño del complejo turístico Playa la Moya

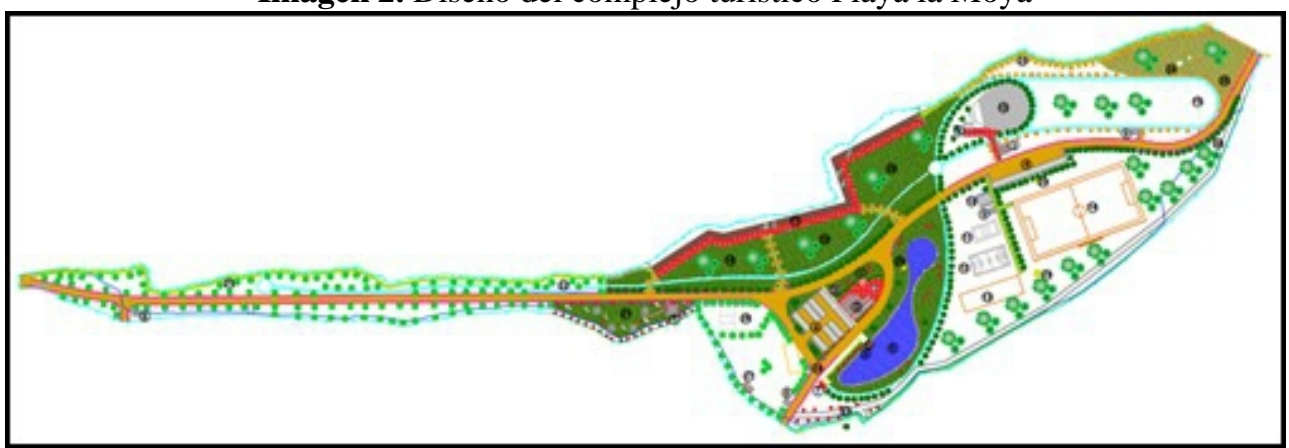

Elaborado por el equipo de investigación

A continuación se presenta la tabla 6 con los emprendimientos y la numeración de ubicación en el mapa.

Tabla 6. Emprendimientos numerados en el mapa

\begin{tabular}{|c|c|c|}
\hline $\begin{array}{c}\text { ESPACIOS DE ENCUENTRO } \\
\text { COMÚN/RECREACIÓN }\end{array}$ & SERVICIOS & OTROS \\
\hline 5 área de embarque de canoas & 1 administración & 23 vías de acceso \\
6 laguna artificial & 2 restaurante & viviendas \\
11 cancha vóley & 3 batería sanitaria & 24 vía principal \\
12 cancha básquet & 4 parqueaderos & 25 caminera \\
13 cancha pelota de mano & 7 área alquiler & peatonal \\
14 estadio & bicicletas & 29 vertiente de \\
15 concha acústica & 9 almacén & agua \\
16 área de escalada & & 30 cisterna de \\
17 muelle & & captación \\
18 área de juegos infantiles & & garita de ingreso \\
19 gimnasio al aire libre & & de agua \\
20 área de juegos lúdicos & & 10 baterías \\
21 área de picnic & & sanitarias y \\
22 ciclo vía & & vestidor \\
26 área de camping & & 28 parqueadero \\
27 caballerizas y pastizales & & buses \\
\hline
\end{tabular}

Elaborado por: equipo de investigación

\section{Discusión}

Esta investigación revela importantes resultados acerca del turismo comunitario en el Ecuador. Los atributos con los que cuenta esta modalidad de turismo han dado paso para contribuir en el posicionamiento de la marca turística del país. La investigación evidencia que el turismo comunitario es de elevado interés para los turistas nacionales; se identificó que el 34\% de los visitantes en un rango de 36 a 45 años son personas económicamente activas que están en la disposición de realizar turismo comunitario, esta variable se presenta como una oportunidad y estrategia de competitividad para diseñar actividades enfocadas hacia este segmento de mercado que estarán en la capacidad de aprovechar oportunamente las implementaciones, 
mientras que en un análisis comparativo con la demanda turística a nivel internacional es posible mencionar el caso de Puerto Rico, en donde las personas que realizan esta actividad se encuentra en una edad a partir de los 50 años representado por el mayor porcentaje de $43.9 \%$ y son personas jubiladas, esta es una variable problemática ya que este segmento de mercado muestra muy poca disposición para la visita y la realización de diferentes actividades que no son apropiadas para su disfrute (Villalobos et al., 2009).

Se identificó que el 92\% de los encuestados conoce la parroquia Guasuntos de los cuales el $83 \%$ ha visitado la parroquia, variable que se presenta como oportunidad para captar un mercado potencial, ya que del total de las encuestas aplicadas el 96\% le gustaría que se implementara el Complejo Turístico Comunitario.

En un estudio de demanda turística internacional, Villalobos et al. (2009), mencionan que 81.5\% de los turistas internacionales que visitan Puerto Rico les gustaría conocer espacios de carácter ambiental promovidos por una educación de protección y conservación de los recursos naturales, esto se debe a que el país oferta la modalidad de turismo de naturaleza y está enfocado en captar ese segmento de mercado; mientras que en el caso del complejo turístico comunitario "Playa de la Moya”, los visitantes han elegido que la implementación sea de carácter recreativo con una aceptación de 61\% y ambientalista con una aceptación del 39\%, esto se debe a que el Ecuador se presenta con una oferta de turismo comunitario, lo cual permite identificar que aunque el país se oferta a nivel internacional tambien motiva la movilidad y visita de turistas nacionales en un alto porcentaje, convirtiéndolo en un sector importante para la generación de recursos económicos y en una oportunidad para identificar otros segmentos de mercado y diseñar actividades para su disfrute.

Vizuete et al. (2015) mencionan que las principales actividades a desarrollarse en el producto turístico comunitario "Pistishi-Nariz del Diablo" con mayor aceptación por los visitantes encuestados son 17\% cabalgata, 19\% senderismo, 16\% Biking, 5\% camping, 17\% fotografía turística y 12\% recorrido en granjas que son actividades que integran la oferta turística, mientras que en el complejo turístico "Playa de la Moya” se diseñaron varias actividades acorde al territorio, entre las cuales se pudo evidenciar algunas con un porcentaje alto de aceptación: ciclismo 23\%, cabalgata 14\%, camping $18 \%$ y escalada $29 \%$, adicionalmente como un aporte a la comunidad y para fortalecer la oferta turística se diseñaron espacios de encuentro común con alto nivel de aceptación como: concha acústica 24\%, parqueadero 20\%, comedor popular $13 \%$, canchas deportivas $16 \%$ y espacios verdes $22 \%$, estos espacios también promueven la generación de otras modalidades de turismo beneficiando así al territorio. 
En un análisis que aborda el segmento del turismo rural en España, se determina que más de la cuarta parte de los turistas realizan esta actividad en época de veraniega, concentrando sus visitas en determinados meses (julio y agosto) (García, 1995); mientras que en el caso del complejo turístico "Playa de la Moya" como resultado de las encuestas aplicadas a los turistas nacionales se identificó que no existe un mes o meses de preferencia para realizar esta modalidad de turismo, sin embargo se identificaron días específicos que les gustaría realizar esta actividad: domingo 46\%, sábado $31 \%$ y viernes $23 \%$, esto permite inferir que se recibirán visitantes todo el año.

La demanda turística es analizada a partir de los consumidores actuales y potenciales que desean, pueden y están dispuestos a disfrutar el conjunto de facilidades, atractivos, actividades, bienes y servicios turísticos, este análisis se desarrolla en función del precio y su rentabilidad, identificando la importancia de generar rubros económicos para medir la eficacia de un proyecto de turismo (Rigol et al., 2009), sin embargo para el turismo comunitario no se puede establecer un precio que genere rentabilidad económica como único fin, la inversión en proyectos de desarrollo parte del apoyo de instituciones gubernamentales, no gubernamentales y como inversionista principal la comunidad, aportando como contraparte del proyecto, no con el objetivo de recuperar la inversión, pero si con el de dotar a la comunidad con un espacio adecuado y dispuesto para la generación y desarrollo de iniciativas productivas, aportando al desarrollo del capital humano y a mejorar la calidad de vida de la población, ya que la construcción de un producto turístico también promueve el mejoramiento de la infraestructura básica de las comunidades; sin embargo en este estudio se pudo identificar, mediante la entrevista, que el 67\% de los visitantes están dispuestos a realizar un pago mínimo para el ingreso al complejo turístico.

En un análisis de la segmentación de la demanda turística española las principales fuentes de información turística que se identificaron fueron: experiencia propia, recomendación del agente de viajes, noticias, libros, información general, folletos y catálogos de ofertas turísticas (González \& Molina, 2007), mientras que en el caso de la segmentación de los turistas nacionales de Ecuador, los mismos mencionaron que les gustaría informarse de estas actividades por medio de publicidad 42\%, internet 32\%, material publicitario impreso 21\% y anuncios en radio 5\%, lo que evidencia que los turistas nacionales tienen preferencia de informarse por los medios tradicionales.

\section{Conclusion}

El estudio realizado permitió identificar la demanda insatisfecha, el perfil del turista y el segmento de mercado enfocados en conocer los gustos y 
necesidades de los visitantes, con el fin de diseñar un producto turístico destinado al aprovechamiento de los visitantes potenciales, el análisis permitió identificar que el 34\% de los turistas están en los rangos de 36 a 45 años que son personas económicamente activas y tienen la disposición de realizar diversas actividades, han elegido que la implementación sea de carácter recreativo con una aceptación de 61\% y ambientalista con una aceptación del 39\%, esto se debe a que el Ecuador se presenta con una oferta de turismo comunitario, los días específicos que les gustaría realizar esta actividad: domingo 46\%, sábado $31 \%$ y viernes $23 \%$, lo que permite determinar que el complejo tendrá visitantes la mayor parte del año y que los turistas nacionales tienen preferencia de informarse por los medios de publicidad tradicionales como internet, material publicitario impreso $\mathrm{y}$ anuncios en radio.

El producto turístico comunitario Complejo Turístico Playa la Moya es un espacio que ofrece la oportunidad de captar iniciativas productivas generadas por la población permitiendo así desarrollar el capital humano del territorio y contribuyendo al mejoramiento de las economías locales. Este espacio también permite la integración de la población para realizar diversas actividades que aportan al fortalecimiento común.

Se plantearon varias preguntas como: edad, si ha visitado Guasuntos, si les gustaría la implementación del complejo turístico, el fin de implementación complejo turístico, actividades, días de visita y medios de publicidad, que fueron tomadas como indicadores de la rentabilidad y accesibilidad para el segmento de mercado y oportunidades para el aprovechamiento de los recursos del territorio, determinando así estrategias para la venta del mismo.

\section{References:}

1. De La Cruz, D. P. (2007). El turismo comunitario como alternativa para combatir la pobreza: el caso de la Federación Plurinacional de Turismo Comunitario del Ecuador. Mujeres indígenas, territorialidad y biodiversidad en el contexto latinoamericano, p. 213.

2. De La Torre, S. (2010). Turismo comunitario, ¿otro sueño inalcanzable? Polémika, vol. 5, no 1.

3. FEPTCE. (2010). Federación Plurinacional de Turismo Comunitario del Ecuador. (Online). www.feptce.org/images/stories/contenido/ pdfs/estadistico-feptce.pdf (consultado mayo 11 del 2016).

4. García, R. (1995). Fuentes. El turismo rural en España: especial referencia al análisis de la demanda.

5. González, P. R. \& Molina, Ó. (2007). La segmentación de la demanda turística española. Metodología de encuestas, vol. 9, no 1, p. 57-92. 
6. Gudynas, E. \& Acosta, A. (2011). La renovación de la crítica al desarrollo y el buen vivir como alternativa. Utopía y praxis latinoamericana, vol. 16, no 53.

7. Guzmán, T. J. \& Cañizares, S. M. (2009). Turismo comunitario y generación de riqueza en países en vías de desarrollo. Un estudio de caso en el salvador1. REVESCO: revista de estudios cooperativos, no 99, p. 85.

8. Lledó, P \& Rivarola, G. (2007). Gestión de proyectos. Pearson Educación.

9. MAE. (2001). Ministerio del Ambiente, Ecociencia, UICN La Biodiversidad del Ecuador, Informe 2000. C. Josse (ed). Ministerio del Ambiente, Ecociencia, UICN. Quito

10. MCPEYC. (2014). Ministerio Coordinador de Producción, Empleo y Competitividad. Matriz productiva. (Online).

11. http://www.produccion.gob.ec/

12. Ministerio de Educación (2011). Plan nacional del Buen Vivir o Sumak Kawsay.

13. Mintur (2009). Ministerio de Turismo. (Online).www.turismo.gob. ec http://www.usfq.edu.ec/publicaciones/polemika/Documents/polemika 005/polemika005_006_articulo003.pdf

14. Rigol, L. M. (2009). Conceptualización de la demanda turística. Ciencias Holguín (Online). 2009, XV (Enero-Marzo): (Fecha de consulta: 3 de febrero de 2017). Disponible en:<http://www.redalyc.org/articulo.oa?id=181517987002> ISSN

15. Ruiz, E., et al. (2008). Turismo comunitario en Ecuador. Comprendiendo el community-based tourism desde la comunidad. Pasos. Revista de turismo y patrimonio cultural, vol. 6, no 3, p. 399418.

16. Senplades, S. N. (2014). Plan Nacional del Buen Vivir.

17. UNESCO (2015). Listas del patrimonio cultural inmaterial y el Registro de mejores prácticas de salvaguardia. http://www.unesco.org/culture/ich/es/listas

18. Vázquez Alvarado, J. M. \& Damián, M. Á. (2015). Estimación empírica de elasticidades de oferta y demanda. Revista mexicana de ciencias agrícolas, vol. 6, no 5, p. 955-965.

19. Villalobos-Céspedes, D.; Galdeano-Gómez, E.; \& Tolón-Becerra, A. (2009). Demanda turística internacional por el turismo naturaleza en Costa Rica: Indicadores socio-demográficos y de condición de viaje. Revista de Ciencias Económicas, vol. 27, no 2.

20. Vizuete, D. C., Flores, C., Zurita, M., \& Román, G. (2015). Turismo rural comunitario un medio para la acción publica para el ser humano y la vida. Estudio de caso: producto turístico rural 
comunitario, Pistishi- Nariz del Diablo, en Ecuador. European Scientific Journal, 11(14).

\section{Anexos}

Anexo $N^{0}$ 1. Entrevista dirigida para los turistas nacionales

\section{Estimado/a:}

Agradecemos su colaboración en esta encuesta, pues la información brindada será de gran ayuda para la implementación del Complejo Turístico "Playa La Moya” - parroquia Guasuntos - cantón Alausí - provincia de Chimborazo.

\section{Datos Generales}

\section{Género}
a. Masculino
b. Femenino

2. ¿En cuál de los siguientes rangos de edad se encuentra usted?
a. 15 - 25años
b. 26 - 35años
c. 36 - 45años
d. 46 - 55años
e. Mayor a 55años

3. ¿Conoce usted o ha escuchado acerca de la parroquia Guasuntos del cantón Alausí?
a. Sí
b. No

4. ¿Le interesaría visitar la parroquia Guasuntos?
a. Sí
b. No

5. ¿Conoce usted o ha escuchado acerca de la Playa La Moya ubicada en la parroquia Guasuntos?
a. Sí
b. No

6. ¿Le gustaría que se implemente un Complejo Turístico en la Playa La Moya?
a. Sí
b. No

7. ¿Con qué fin le gustaría que se implemente el Complejo Turístico “Playa La Moya”?
a. Educativos
b. Recreativos
c. Ambientales
d. Otros (especifique)

8. ¿Qué actividades le gustaría que se implemente en el Complejo Turístico "Playa La Moya”?
a. Ciclismo
b. Cabalgata
c. Camping
d. Escalada
e.Otras (especifique)

9. ¿Qué espacios de encuentro común adicionales le gustaría que se implemente en el Complejo Turístico "Playa La Moya”?
a. Concha acústica
b. Parqueadero
c. Comedor popular
d. Espacios verdes
e. Canchas deportivas
f. Espacios verdes
g. Otros (especifique)

10. ¿En qué días visitaría con mayor frecuencia el Complejo Turístico "Playa La Moya”?
a. Lunes
b. Martes
c. Miércoles
d. Jueves
e. Viernes
f. Sábado
g. Domingo 
11. ¿En qué horarios a usted le gustaría visitar el Complejo Turístico "Playa La Moya”?
a. 7 am - $6 \mathrm{pm}$
b. 8 am - 6 pm
c. 9 am -6 pm
d. Otros (especifique)

12. ¿Estaría dispuesto/a a pagar un costo mínimo de ingreso al Complejo Turístico "Playa La Moya”, para garantizar la sostenibilidad económica del mismo?
a. Sí
b. No

13. ¿Por qué medio le gustaría informarse acerca de las actividades y promociones que genere el Complejo Turístico "Playa La Moya”?
a. Internet
b. Radio
c. $\mathrm{Tv}$
d. Prensa escrita
e. Material publicitario impreso
f. Otros (especifique) 